\title{
ICBA Treatment of Chronic Un-healing Lesion in a Femoral DVT Patient-A Hypothesis of the Mechanism of Action
}

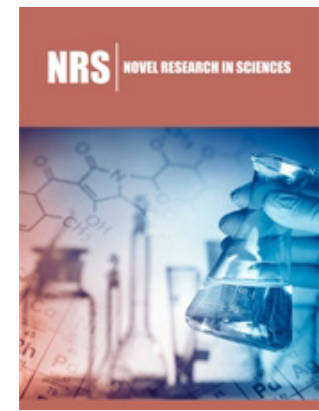

*Corresponding author: Arkady Kotlyar, Pain Clinic, Israel

Submission: 眥June 06, 2019

Published: 䐬October 16, 2019

Volume 1 - Issue 4

How to cite this article: Arkady K.ICBA Treatment of Chronic Un-healing Lesion in a Femoral DVT Patient-A Hypothesis of the Mechanism of Action.Nov Res Sci.2(2). NRS.000535.2019.

DOI: 10.31031/NRS.2019.2.000535

Copyright@ Arkady Kotlyar, This article is distributed under the terms of the Creative Commons Attribution 4.0 International License, which permits unrestricted use and redistribution provided that the original author and source are credited.

\author{
Arkady Kotlyar*
}

Pain Clinic, Israel

\begin{abstract}
Introduction: DVT is a major, preventable cause of death worldwide. Ultrasound (US) is universally accepted as the first-line imaging procedure for DVT diagnosis.

Case presentation: Three years ago, a 59-years-old patient detected a painless lesion above the right lateral malleolus. After starting to feel strong, right femoral pain, the patient sought treatment at a community health medical organization (HMO) and was urgently referred to a hospitalization. During a 5-day hospitalization, the patient was diagnosed to suffer from right femoral DVT, according to the venous Doppler US results. The patient was treated with clot-dissolving drugs. Upon discharge from the hospital, the patient was instructed to continue a clot-dissolving drug and to followed-up in an HMO. The patient was instructed to wear an elastic compression stocking on his right leg, and prescribed analgesic and oral antibiotics and. Non-healing of lesion and pain continued for about three years after initial treatment. Since the lesion failed to heal and the pain did not dissipate, the patient decided to attempt acupuncture treatment.
\end{abstract}

Methods: The patient was treated with 12 sessions I Ching Balance Acupuncture (ICBA) and followed up for the next six months.

Results: Lesion healing setup began during the 1st ICBA session. Following the $3^{\text {rd }}$ session, the patient stopped consuming the analgesic. Complete lesion healing occurred after 12 ICBA sessions and persisted throughout the follow-up period.

Conclusion: ICBA treatment successfully affected the setup of the healing process of an unhealing lesion. Additional large-scale studies are necessary to validate the efficacy of ICBA in thrombosis.

Keywords: Complementary and Alternative Medicine (CAM); I ching balance acupuncture; Thrombosis

Abbreviations: BDNF: Brain Derived Neurotrophic Factor; CBC: Complete Blood Count; CDT: CatheterDirected Thrombolysis; CNS: Central Nervous System; DNA: Deoxyribonucleic Acid; DVS: Doppler Or Duplex Venous Scanning; DVT: Deep Vein Thrombosis; ED: Emergency Department; GB: Gallbladder (Acupuncture Meridian); HMO: Health Medical Organization; ICBA: I Ching Balance Acupuncture; IL1R1: Interleukin-1 Receptor-Alpha; IP: Intra-Peritoneal; LR: Liver (Acupuncture Meridian); MD: Medical Doctor; mRNA: Messenger Ribonucleic Acid; NOAC: Novel Oral Anticoagulant; PTS: Post-Thrombotic Syndrome; PO: Per Os; PE: Pulmonary Embolism; SP: Spleen Pancreas (Acupuncture Meridian); ST: Stomach (Acupuncture Meridian); TE: Triple Energizer (Acupuncture Meridian); TCM: Traditional Chinese Medicine; US: Ultrasound

\section{Introduction}

Deep vein thrombosis (DVT) is a major, preventable cause of death worldwide, affecting approximately $0.1 \%$ of the population each year [1]. Femoral DVT is one of two types of thigh or proximal vein thrombosis [2]. This condition is well-known and documented in medical literature. Once a thrombus is formed in a thigh vein, single-vein obstruction induces vascular insufficiency on the distal side of the vein [2]. It results in clinical symptoms such as redness and pain [2]. Therefore, acute unilateral leg pain and/or swelling are common DVT symptoms [3]. Specifically, femoral DVT can lead to recurrent thrombosis and post-thrombotic syndrome (PTS) [4]. PTS is a painful condition that can potentially lead to chronic venous insufficiency, edema, and ulceration [4].

Historically, venography was the gold standard for DVT diagnosis [5]. After venous ultrasound (US) evolved, this became the universally accepted first-line imaging procedure for DVT diagnosis [5]. Doppler or duplex venous scanning (DVS) with a sensitivity of $98.7 \%$ 
and specificity of $100 \%$ has become the standard diagnostic modality for proximal DVT [6]. Standard DVT treatment focuses on reducing immediate complications [7]. Thrombolysis and clot-dissolving drugs reduce the long-term complications of PTS [7]. A minimally invasive endovascular treatment that is used as an adjunct to anticoagulation is catheter-directed thrombolysis (CDT) [8]. CDT lowers the risk of PTS by reducing clot burden and protecting against valvular damage [8]. To date, DVT incidence and risk factors have been poorly characterized [9]. Nevertheless, proximal DVT, including thrombus in the femoral vein, can usually be diagnosed and treated during initial treatment in the hospital Emergency Department (ED) [3].

\section{Case Presentation}

About three years ago, a 59-years-old male Caucasian patient detected a painless lesion above the right lateral malleolus. No lesion healing was observed for a year after the lesion was detected. When strong, right-femoral pain began irradiating to the groin, the patient contacted a physician at his HMO and was urgently referred for hospital treatment. All the analyses performed at the hospital ED, including blood chemistry, CBC, and coagulation, did not reveal any pathological findings. Nonetheless, a venous blood circulation problem was suspected. The patient received a Clexane (Enoxaparin) 80mg intra-peritoneal (IP) injection and was hospitalized for continued treatment in the Internal Medicine Ward. During hospitalization, a venous Doppler ultrasound (US) exam of the legs was performed. The exam revealed right-femoral, deep-vein thrombosis (DVT). The patient was treated with Xarelto (Rivaroxaban) $15 \mathrm{mg}$ per OS (PO) tablets twice a day; Coumadin (Warfarin) 2-5mg PO tablets; and Clexane (Enoxaparin) 80mg daily IP injection, during a week of hospitalization. After a week of treatment in the hospital, the patient was discharged without pain, and Xarelto (Rivaroxaban) 15mg PO tablets were prescribed twice daily for three weeks, and $20 \mathrm{mg} /$ day for six months thereafter (Figure 1). After hospitalization, the patient followed up with an HMO physician for an additional 6 months. Upon recurrence of pain, analgesic (Optalgin, a derivative of Metamizole) 100mg tablets were prescribed. Since no lesion healing was observed for over six months post-hospitalization, the patient was instructed to wear an elastic compression stocking. Subsequently, the HMO physician prescribed oral antibiotics for the patient, to prevent infection of the unhealing lesion. Despite these measures, non-healing of the lesion and upper thigh pain persisted for about three years after the setup, and for about two and a half years post-hospitalization. Thereafter, the patient sought acupuncture treatment.

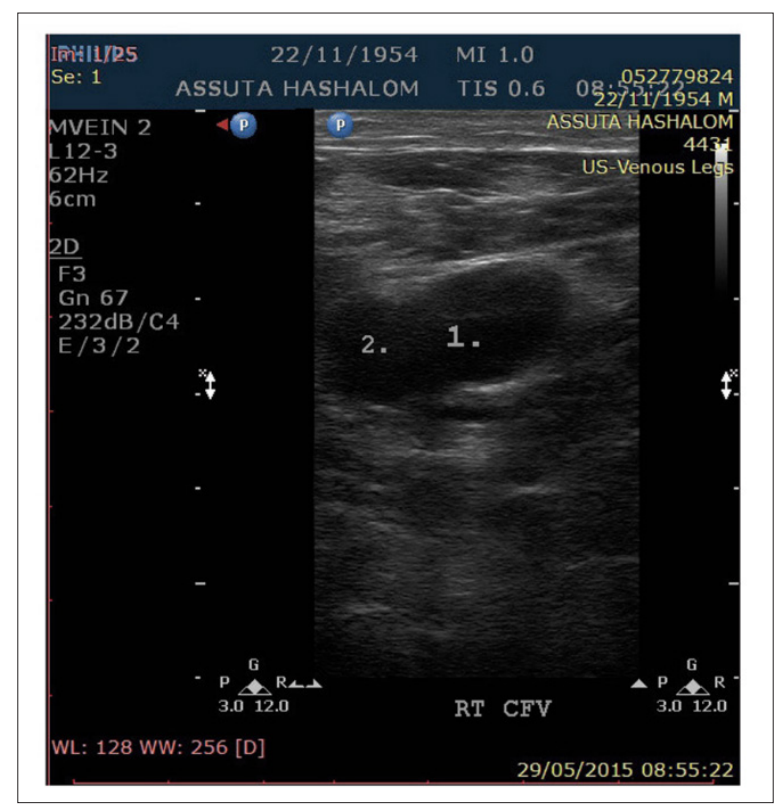

Figure 1: Doppler US the right thigh- 2015 Compressed Femoral Vein, Thrombus

\section{Methods}

\section{Diagnostics and treatment}

One of the patient's chief complaints was an unhealing lesion above the right lateral malleolus, along the GB (Foot Shao Yang) meridian. thus, foot shao yang was defined as a "sick" meridian. The lesion was defined as a local imbalance, which required treatment based on a local balance approach (Figure 2) [10]. In addition, the patient complained of severe pain in the right upper thigh, for which he consumed up to 10 analgesic tablets daily. The pain was localized along the SP (foot tai yin) meridian. However, as opposed to the lesion, the pain resulted from DVT, a derivative of a systemic coagulation disorder. Thrombosis was defined as a global imbalance which required a global balance approach for treatment [10]. ICBA pulse diagnostics were used to confirm the involvement of the diagnosed meridians [11]. the pulses clearly indicated an excess (Shi) of foot tai yin and foot shao yang meridians [11]. After affirming the affected meridians, the five systems of the balance method of i ching acupuncture were employed [11]. System 1 was perfectly suited for local balance treatment of the lesion. Therefore, contralateral ashi TE (hand Shao yang) 5 area was needled. the involvement of foot tai yin and foot shao yang meridians that was 
confirmed by the Chinese pulse diagnostics was the basis for global treatment of the source of pain. considering that hand shao yang 5 did not affect hand shao yang hexagram, it was added to the meridian inter-meridian conversion. thus, it allowed for combining global and local balance approaches without weakening their effects. Table 1 summarizes the used points and treatment laterality.

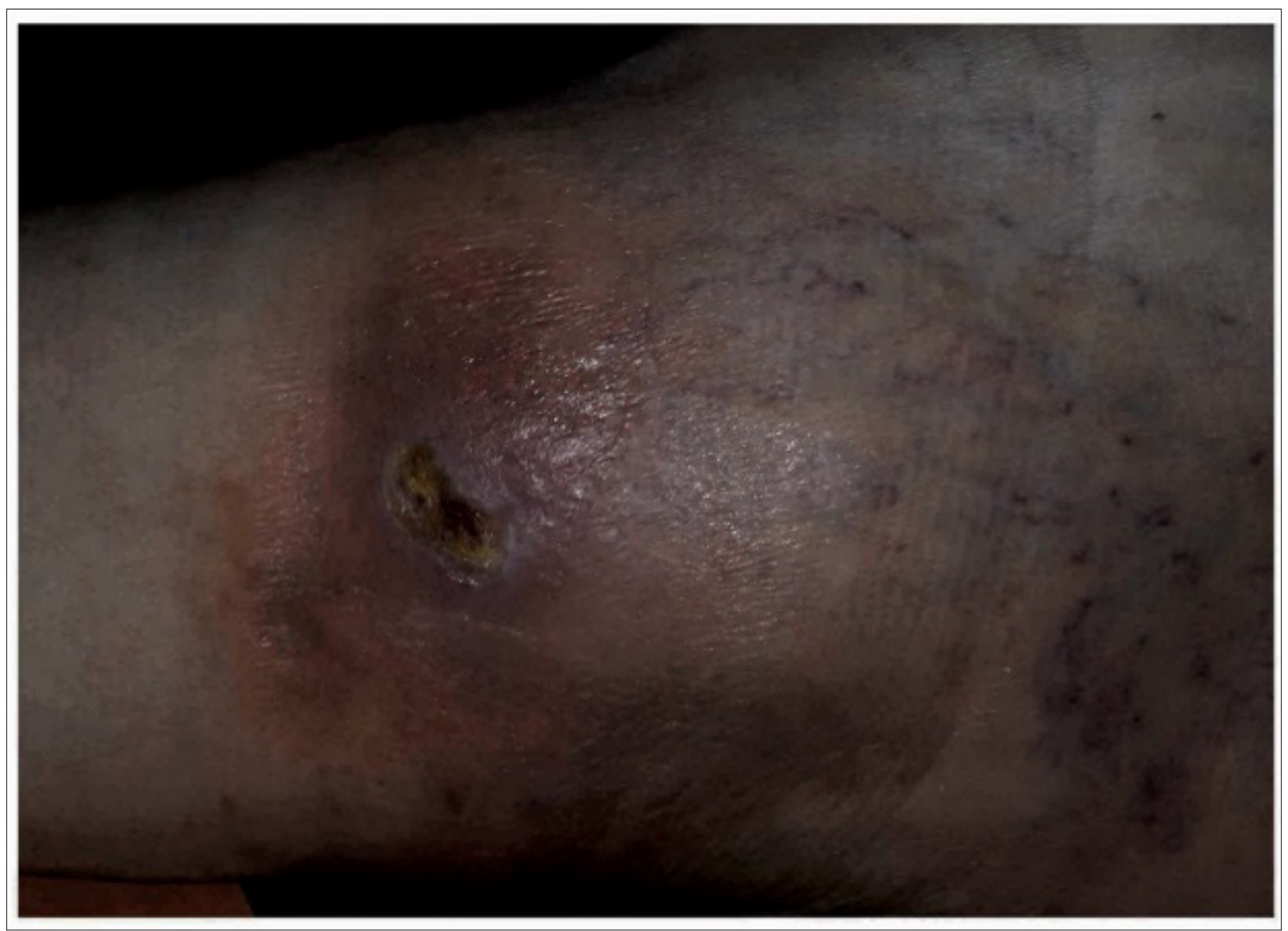

Figure 2: A chronic, un healing lesion.

Table 1: Acupoints used for treatment.

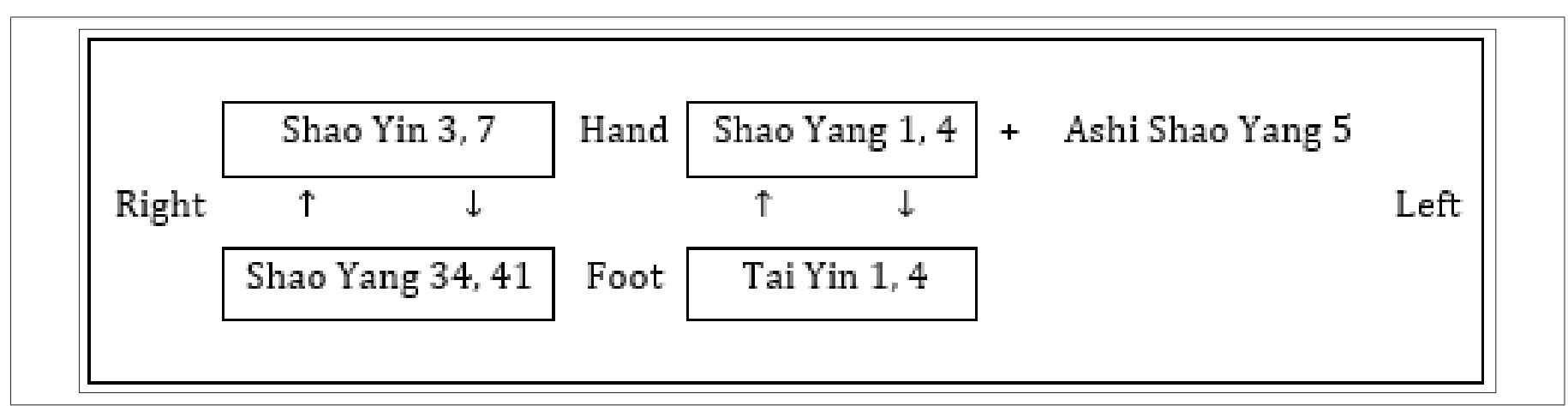

\section{Duration and frequency of ICBA sessions}

The patient was treated with i ching balance acupuncture in two evenly spaced, 60-minute sessions per week. The patient discontinued treatment after 12 sessions, after approximately 6 weeks. After discontinuation of ICBA treatment, follow-up continued for six months.

\section{Results}

Decrease of hyperemia and swelling were observed around the lesion immediately after the $1^{\text {st }} 60 \mathrm{~min}$ ICBA session. Following the $3^{\text {rd }} 60 \mathrm{~min}$ ICBA session, performed 1.5 weeks into the treatment, the patient stopped feeling pain and stopped consuming the analgesic. A complete closing of the lesion occurred 6 weeks after the beginning of treatment, consisting of 12 ICBA $60 \mathrm{~min}$ sessions (Figure 3). After discontinuation of treatment, a further advance in lesion healing was observed during the 3 and 6-month follow-up examinations. The results of doppler US with thigh compression that was performed in 2018 were qualitatively compared with those obtained in 2015. A slight decrease of the thrombus size was detected in 2018 at the 3-months follow-up session after 6-weeks ICBA treatment, compared to its size in 2015 (Figure 4). 


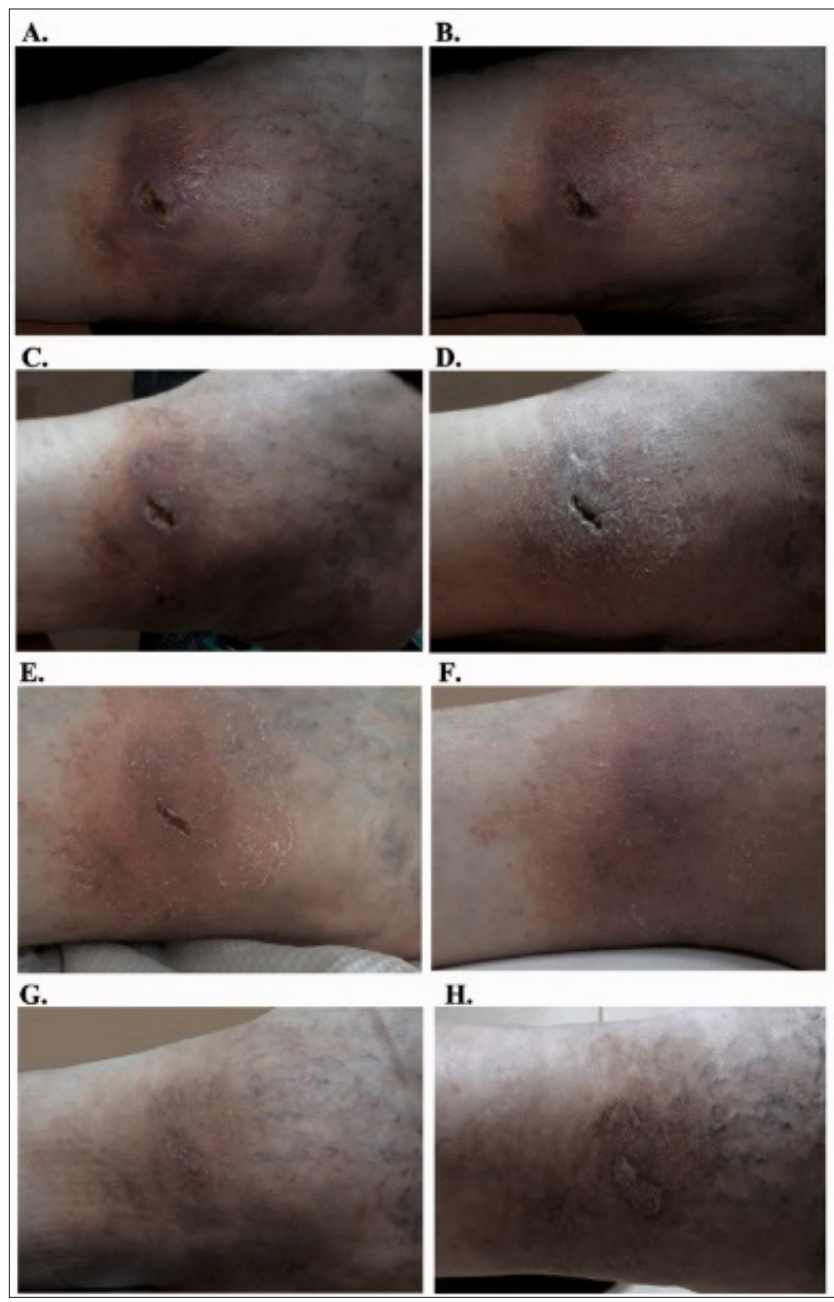

Figure 3: Lesion during acupuncture treatment \& 6 months follow up

A. Before the treatment, B. Immediately post 60 min of the 1 st session, C. Post 3rd session, D. Post 5 th session, E. Post 8th session, F. Post 12th session, G. Post 3 months of follow up, H. Post 6 months of follow up.

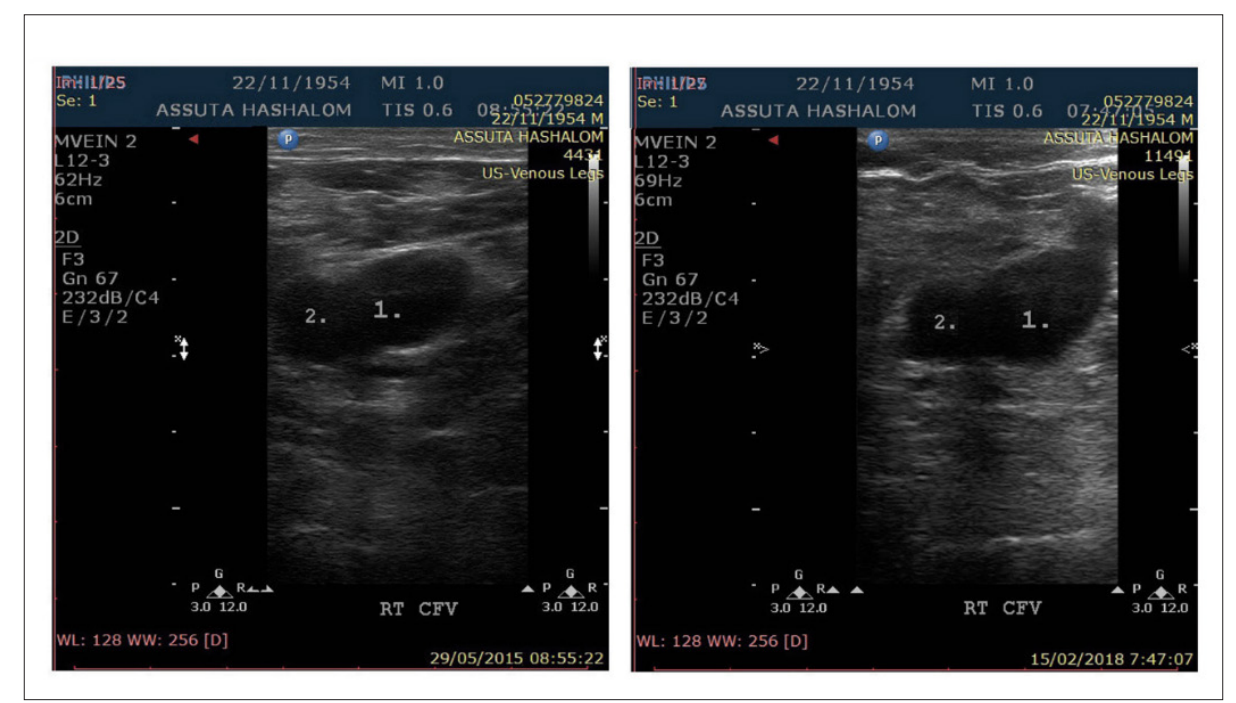

Figure 4: Doppler US with compression of the right thigh - 2015 vs. 2018

A. 2015 , B. 2018

1. Compressed Femoral Vein, 2. Thrombus. 


\section{Discussion}

There are several options for DVT treatment-some already available and others being developed. In his review, Shel long SM named the two main reasons for treatment of proximal DVT, [12] namely prevention of pulmonary embolism (PE), and PTS. Vo et al. [13] describe anticoagulation as the basis for treatment in DVT patients. Therefore, parenteral anticoagulants and oral vitamin $\mathrm{K}$ antagonists have been used for many decades [13]. However recent development of novel oral anticoagulants (NOACs) offers an entire set of new therapeutic options for DVT. Fujino et al. [14] showed that use of NOACs was effective for dissolving small-sized DVT in outpatients in poor condition. A study by Puskas et al. showed that efficient anticoagulant and compressive therapy caused spontaneous recanalization of DVT. In their study, almost complete re-permeabilization was observed after 12 months of treatment [15].

Zwingerman et al. [16] conducted a comprehensive literature review and identified an association between Alanine and Threonine variants with venous thrombosis. De la et al. [17] mention that several reports describe Valine 34 Leucine polymorphism as a protective factor against venous and arterial thrombosis. An attempt to prevent DVT using Traditional Chinese Medicine (TCM) in postoperative bedridden elderly patients was reported by Hou et al. [18]. The results of the study showed that electrical stimulation of Yinlingquan (SP 9), Sanyinjiao (SP 6), Taichong (LR 3) and Zusanli (ST 36) acupoints increased circulation in the lower limb, thus preventing DVT. An additional study by Hou et al. [19] reported that electrical TCM acupoint stimulation showed great potential for preventing symptom less DVT in elderly patients.

The results of the present study show a slight decrease in thrombus size, when comparing the results of the doppler US performed in 2018 with those of 2015. It is conceivable that at least to some extent, the decrease in thrombus size can be explained by spontaneous recanalization. An occurrence of spontaneous recanalization was described by Puskas et al. [15]. Moreover, since no doppler US was performed before ICBA treatment the status and dynamics of thrombosis before ICBA treatment remains unknown. Such a limited number of sessions were not anticipated to completely dissipate a chronic and systemic disorder such as thrombosis. On the other hand, the prompt and consistent effect of ICBA treatment on the chronic, unhealing lesion and on pain was observed from the very beginning of the treatment (Figure 3). A gradual and substantial improvement in the appearance of the chronic, unhealing lesion and dissipation of chronic pain are the two major case findings in this study, which suggest the following:

A. A photo of the 3-year old, unhealing lesion immediately post $1^{\text {st }}$ ICBA session shows decreased swelling and hyperemia around the lesion (Figure 3). This improvement indicated the setup of a healing process. Furthermore, the 3-year old, unhealing lesion continued to heal and become encrusted after the $12^{\text {th }}$ ICBA session.

B. Chronic, 3-year old femoral pain irradiating to the groin completely dissipated after the $3^{\text {rd }}$ ICBA session.
Both findings correspond with the detected decrease in thrombus size throughout the post-treatment follow up period, which was most probably initiated as soon as ICBA treatment began. All the above indicate that healing began as early as during the first 60-minute ICBA session and continued throughout the entire ICBA treatment period. Spontaneous recanalization certainly can occur and this must be considered. However, considering all the above, it seems less plausible that spontaneous recanalization alone can account for complete dissipation of 3 year-lasting pain and an unhealing lesion.

Note that:

a. Despite the recommendation the patient did not wear an elastic compression stoking;

b. The ICBA effect occurred promptly and persisted over the course of 6 weeks of the treatment.

c. The above raises questions about a possible mechanism of ICBA performance, as hypothesized below.

\section{Hypothesized ICBA mechanism of action}

To begin with, based on the similarities between DNA base pairs and Ho Tu Huan He (Yellow River Map) a genetic mechanism of ICBA action is theoretically possible. An association between Alanine and Threonine variants with thrombosis was previously reported [20]. Presumably, needling of certain acupoints on one or several 12-paired acupuncture meridians theoretically affects DNA codons $[16,17]$. To support the hypothesis, Castro-Chavez F reported that I Ching of the oldest emperor of China Fu-Xi precisely depicted the genetic code that exists today. Also, he concluded that the first representation of the 64 codons in modern times is like the first binary I Ching of Fu-Xi. Castro-Chavez concluded that the theoretical importance of representing the complete genetic code as amino acids was revealed through the shape of the double pair of genetic code chromosomes obtained in his work [21].

Shiue et al. [22] analyzed a differential gene expression in the peripheral blood of patients with allergic rhinitis before and after acupuncture treatment, using cDNA microarray. The results of the cDNA microarray analysis showed that the expression of interleukin-1 receptor-alpha (IL1R1) in peripheral blood was significantly decreased at 2-hours, 24-hours, and 4-weeks after acupuncture treatment. Their results also suggest that acupuncture treatment affected the balance of pro- vs. anti-inflammatory cytokines. Lin et al. [23] have reviewed the recent findings and therapeutic implications of acupuncture-induced activation of brain-derived neurotrophic factor (BDNF) and it signaling pathway. A review by Chavez et al. [24] showed that acupuncture exerts beneficial effects on ischemic stroke through modulation of different mechanisms originating in the CNS.

Jiang et al. [25] suggested that DNA methylation and histone modifications of BDNF are the epigenetic mechanisms of the antidepressant effect of acupuncture. Liu et al. [26] reviewed current methods of skeletal muscle tissue repair and regeneration. They indicated that applying acupuncture with low-frequency electrical 
stimulation (Acu-LFES) for treatment of diabetic myopathy and muscle loss induced by chronic kidney disease, showed functional muscle improvement. The underlying mechanism of the improvement includes activation of M2 macrophages and reversing mRNA expression levels of the E3 ubiquitin ligase atrogin-1. Based on the above, a genetic mechanism of ICBA action is theoretically possible. Considering the data obtained in the present case report,
ICBA might cause production of corresponding amino acids by modifying certain codons. The presumed effect of acupuncture points needled for a global inter-meridian conversion, subsequent codon modifications, and translation to the corresponding amino acids is summarized in Table $2 \& 3$. However, the ICBA mechanism of action proposed above is still only a hypothesis. It is conceivable that ICBA triggers other not yet explored mechanisms.

Table 2: DNA nucleotide code to I Ching equivalent translation.

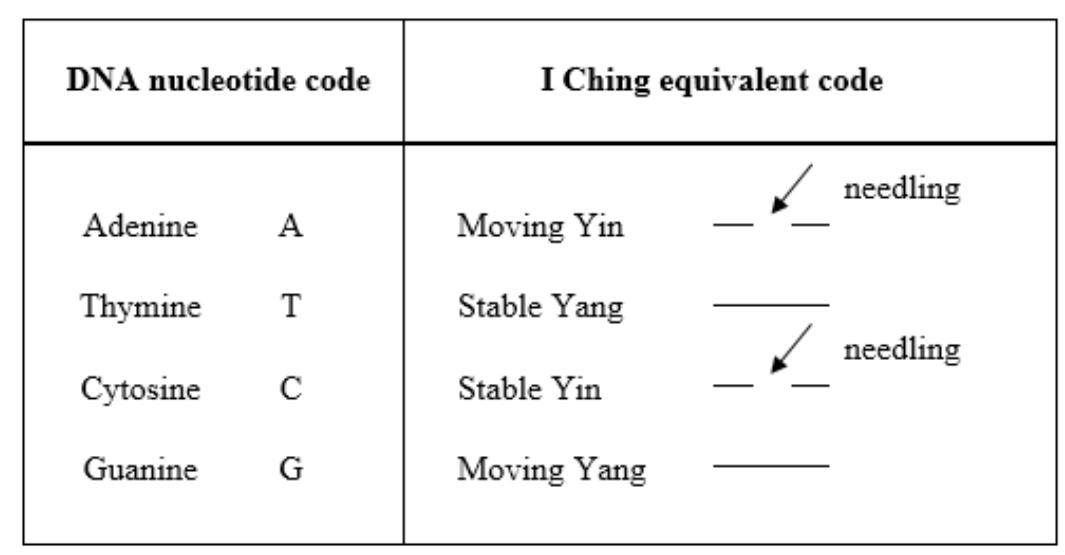

Table 3: Summary of the effect of acupuncture points needled for global inter-meridian conversion, subsequent codon modifications and translation to corresponding amino acids.

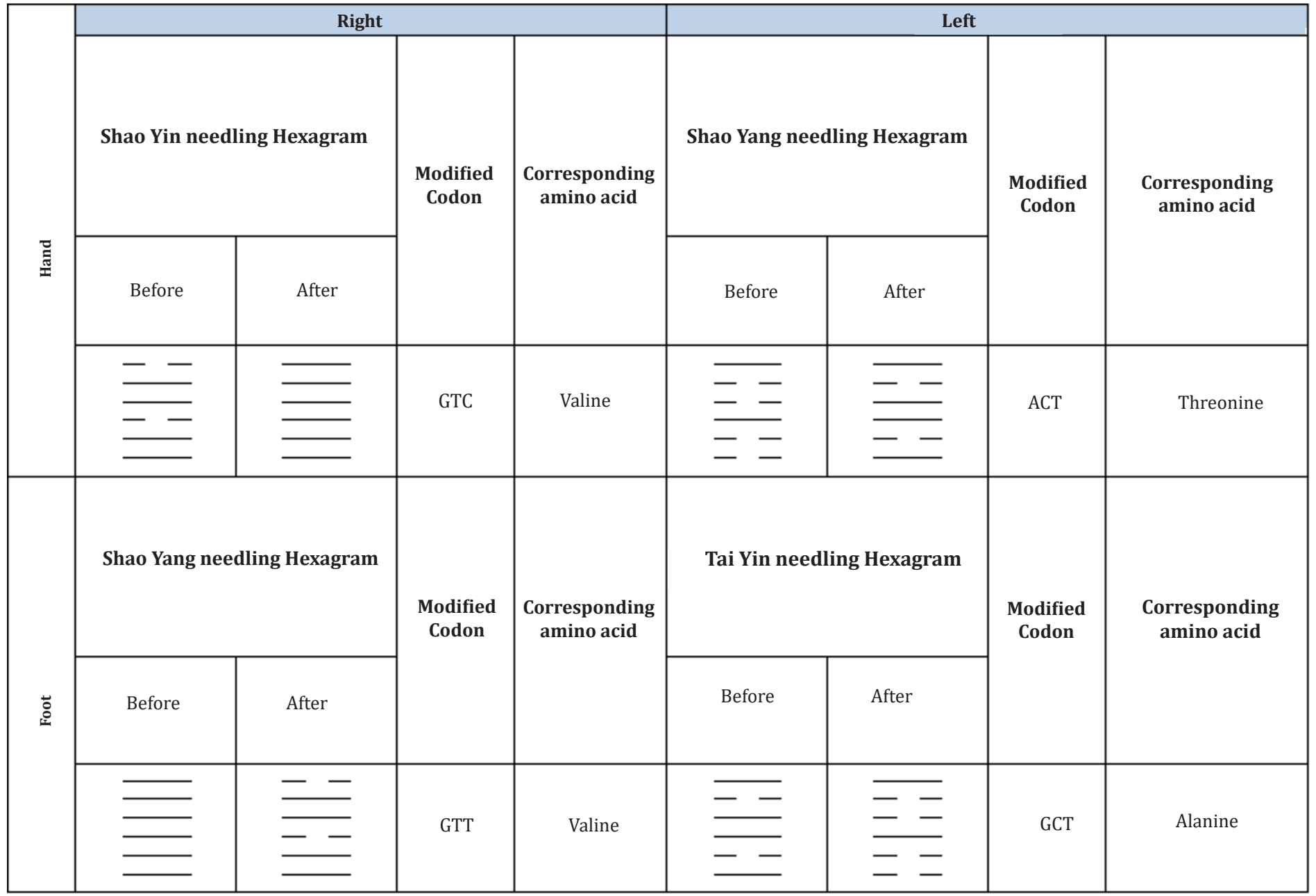


The scientific importance of the case report presented here is certainly minor. Yet, it adds another tiny cut to previously reported ICBA effects. On the other hand, the prompt therapeutic response described here offers several directions for future ICBA research. Further investigation of DNA-ICBA similarities described in scientific literature and the effect of ICBA treatment on amino acids should also be considered.

\section{Conclusion}

The present study is one of the first to demonstrate the effects of ICBA treatment on a chronic, unhealing lesion in a femoral DVT patient. ICBA treatment successfully affected the setup of the healing process of an unhealing lesion. Healing began during the first 60-minute ICBA session and continued throughout the treatment period. The DNA element of the ICBA mechanism cannot be claimed based on the discussed case alone. This is not more than a hypothesis. Comprehensive studies are needed in order to investigate the ICBA mechanism, and large-scale, focused studies should be conducted to explore the genetic aspect of ICBA effects.

\section{Acknowledgement}

The author sincerely appreciates the courtesy of Dr. Eran Weinmann (MD) in sharing his knowledge and experience. Dr. Weinmann assisted in interpreting the results of this work with great interest. Dr. Eran Weinmann is the head of the Department of Vascular Surgery at Medical Center Kaplan.

\section{References}

1. Kesieme E, Kesieme C, Jebbin N, Irekpita E, Dongo A (2017) Deep vein thrombosis-a clinical review. J Blood Med 2: 59-69.

2. Ro A, Kageyama N, Mukai T (2017) Pathophysiology of venous thromboembolism with respect to the anatomical features of the deep veins of lower limbs-A Review. Ann Vasc Dis 10(2): 99-106.

3. Kitchen L, Lawrence M, Speicher M, Frumkin K (2016) Emergency department management of suspected calf-vein deep venous thrombosis: a diagnostic algorithm. West J Emerg Med 17(4): 384-390.

4. Robertson L, McBride O, Burdess A (2016) Pharmaco mechanical thrombectomy for iliofemoral deep vein thrombosis. Cochrane Database Syst Rev 4(11): CD011536.

5. Palareti G, Schellong S (2012) Isolated distal deep vein thrombosis-what we know and what we are doing. J Thromb Haemost 10(1): 11-19.

6. Henry JC, Satiani B (2014) Calf muscle venous thrombosis. A review of the clinical implications and therapy. Vasc Endovasc Surg 48(5-6): 396401.

7. Watson L, Broderick C, Armon MP (2016) Thrombolysis for acute deep vein thrombosis. Cochrane Database Syst Rev 23(1): CD002783.

8. Fleck D, Albadawi H, Shamoun F, Knuttinen G, Naidu S, et al. (2017) Catheter-directed thrombolysis of deep vein thrombosis: literature review and practice considerations. Cardiovasc Diagn Ther 7(Suppl 3): S228-S237.

9. Repella TL, Lopez O, Abraham CZ, Azarbal AF, Liem TK, et al. (2018) Characterization of profunda femoris vein thrombosis. J Vasc Surg Venous Lymphat Disord 6(5): 585-591.
10. Kotlyar A (2017) Straightforwardness, universality and effectiveness of the balance method of i Ching acupuncture. Medical Acupuncture 29(2): 94-104.

11. Tan R (2007) Acupuncture 1, 2, 3. ISBN-0975941232, 9780975941232.

12. Shellong SM (2007) Distal DVT: worth diagnosing? Yes. Journal of Thrombosis and Haemostasis 5(Supp 1): 51-54.

13. Vo T, Vazquez S, Rondina MT (2014) Current state of anticoagulants to treat DVT. Curr Cardiol Rep 16(3): 463.

14. Fujino T, Yamazaki Y, Yamazaki A, Kabuki T, Kiuchi S, et al. (2015) Efficacy of dabigatran for dissolving DVT in outpatients with deteriorated general condition. Int Heart J 56(4): 395-399.

15. Puskas A, Balogh Z, Hadadi L, Imre M, Orbán E, et al. (2007) Spontaneous recanalization in deep venous thrombosis: a prospective duplex ultrasound study. Int Angio 26(1): 53-63.

16.Zwingerman N, Medina A, Kassam I, Michael D, Pierre E, et al. (2017) Sex-specific effect of CPB2 Ala147Thr but not Thr325Ile variants on the risk of venous thrombosis: A comprehensive meta-analysis. PLoS One 26:12(5): e0177768.

17. De RG, Tàssies D, Espinosa G, Monteagudo J, Bové A, et al. (2009) Factor XIII-A subunit Val34Leu polymorphism is associated with the risk of thrombosis in patients with antiphospholipid antibodies and high fibrinogen levels. Thromb Haemost 101(2): 312-316.

18. Hou L, Chen C, Xu L, Yin P, Peng W (2013) Electrical stimulation of acupoint combinations against deep venous thrombosis in elderly bedridden patients after major surgery. JTCM 33(2): 187-193.

19. Hou LL, Yao LW, Niu QM, Xu L, Yu QH, et al. (2013) Preventive effect of electrical acupoint stimulation on lower-limb thrombosis: a prospective study of elderly patients after malignant gastrointestinal tumor surgery. Cancer Nurs 36(2): 139-144.

20. Roosen F (2007) I ching DNA and acupuncture. The Euro J Integ East West Med 2(5): 19-23.

21. Castro-Chavez F (2012) Defragged binary I ching genetic code chromosomes compared to nirenberg's and transformed into rotating 2D circles and squares and into a 3D 100\% symmetrical tetrahedron coupled to a functional one to discern start from non-start methionines through a stella octangula. J Proteome Sci Comput Biol 1: 3.

22. Shiue HS, Lee YS, Tsai CN, Hsueh YM, Sheu JR, et al. (2008) DNA microarray analysis of the effect on inflammation in patients treated with acupuncture for allergic rhinitis. J Altern Complement Med 14(6): 689-698.

23. Lin D, De I, Lin L, Zhou SF, Borlongan CV, et al. (2014) The neuroprotective role of acupuncture and activation of the BDNF signaling pathway. Int J Mol Sci 15(2): 3234-3252.

24. Chavez LM, Huang SS, Donald I, Lin JG, Lee YC, et al. (2017) Mechanisms of acupuncture therapy in ischemic stroke rehabilitation: a literature review of basic studies. Int J Mol Sci 18(11): 2270.

25. Jiang H, Zhang X, Lu J, Meng H, Sun Y, et al. (2018) Antidepressant-like effects of acupuncture-insights from DNA methylation and histone modifications of brain-derived neurotrophic factor. Front Psychiatry 9: 102.

26. Liu J, Saul D, Boker KO, Ernst J, Lehman W, et al. (2018) Current methods for skeletal muscle tissue repair and regeneration. Biomed Res Int 18: 1984879. 